\title{
First do no harm: changing culture surrounding ceilings of treatment in end-of-life care
}

\author{
Authors: S McNeill, ${ }^{1}$ E Toner, ${ }^{1}$ S Caskey, ${ }^{1}$ AM Marley, ${ }^{2}$ S Guy ${ }^{2}$ and P Elliott ${ }^{3}$
}

\section{Aims}

The NICE Quality Standard for end-of-life care for adults $(2011)^{1}$ provides a far-reaching vision of how high quality endof-life care should appear. Unfortunately, patients approaching the end of life may receive inconsistent care as their condition deteriorates. Failure of the team providing normal medical care to communicate patients' wishes, and clearly document a personalised care plan for current and future support and treatment, can expose patients to burdensome medical investigations and increase anxiety among nursing staff and 'on call' medical teams, should an acute deterioration occur outside of normal working hours.

We sought to determine whether the introduction of a preemptive 'ceiling of treatment' (COT) form would lead to improved discussion and documentation of prognosis and appropriate treatment options in the context of an end-of-life trajectory.

\section{Methods}

Two online survey tools were administered to examine medical and nursing staff experience of caring for patients approaching the end of life. Before and after the introduction of the COT form, inpatient medical records were reviewed for documented decisions regarding ceilings of treatment. Education was delivered to local units to promote the importance of early shared decision making and prognostic conversations in end-of-life care.

\section{Results}

Ninety percent of doctors and $73 \%$ of nurses have witnessed patients undergoing treatments they consider to be futile or burdensome. Seventy-one percent of doctors and $77 \%$ of nurses have been left unsure what active interventions a patient is still considered for. After educational events and the addition of the COT form, the number of patients with a clear COT identified has risen from $12 \%$ to $53 \%$, while those patients documented as DNACPR have had improved COT identification from 35\% to $92 \%$.

Authors: ${ }^{1}$ Regional Adult Respiratory Centre, Belfast City Hospital; ${ }^{2}$ Mater Infirmorum Hospital, Belfast; ${ }^{3}$ Royal Victoria Hospital,

Belfast

\section{Conclusions}

Structural reform at hospital level is vital to enable optimum end-of-life care and we hope the introduction of 'ceiling of treatment' forms coupled with education, will provide a clearer direction for hospital staff as to what is important as patients approach end-of-life care.

\section{Conflict of interest statement}

None

\section{Reference}

1 National Institute of health and Care Excellence. End of life care for adults: quality standard [QS13]. NICE: 2011. 